\title{
Caracterización morfológica de dos genotipos de berenjena (Solanum melongena) cultivados en invernadero en Costa Rica
}

\author{
Cristina Arguedas-García' \& José Eladio Monge-Pérez² \\ 1. Investigadora independiente, San Sebastián, San José, Costa Rica; cris31oct@gmail.com \\ 2. Estación Experimental Agrícola Fabio Baudrit Moreno y Sede de Guanacaste, Universidad de Costa Rica, Costa Rica; \\ jose.mongeperez@ucr.ac.cr
}

Recibido 01-III-2017 • Corregido 20-VI-2017 • Aceptado 07-VII-2017

\begin{abstract}
Morphological characterization of two eggplant (Solanum melongena) genotypes grown under greenhouse conditions in Costa Rica. Eggplant is a vegetable with innovative potential for Costa Rican market. A morphological characterization was conducted of two eggplant genotypes (JMX-292 and JMX-1099) grown under greenhouse conditions in Alajuela, Costa Rica. The qualitative variables evaluated were growth habit, leaf width, leaf spines, corolla color, fruit length, fruit calyx spines, fruit color at commercial maturity stage, and seed quantity. The quantitative variables evaluated were leaf width $(\mathrm{cm})$, number of spines per leaf, fruit length $(\mathrm{cm})$, number of spines per calyx, and number of seeds per fruit. There were significant differences between genotypes for number of spines per leaf $(0,04$ for JMX-1099 vs 1,44 for JMX-292), fruit length (15,50 vs $13,12 \mathrm{~cm}$, respectively), number of spines per calyx $(0,20$ vs 3,48 , respectively), and number of seeds per fruit (199,35 and 631,58, respectively). Both genotypes showed innovative market characteristics, like the white fruit color of JMX-292, as well as the low number of spines per leaf and per calyx and the low number of seeds per fruit of JMX-1099, so these genotypes could be interesting choices for growers.
\end{abstract}

Key words: fruit color, quantity of spines per leaf, quantity of spines per calyx, number of seeds per fruit.
RESUMEN: La berenjena es un cultivo con potencial de innovación en el mercado costarricense. Se realizó una caracterización morfológica de dos genotipos de berenjena (JMX-292 y JMX-1099) cultivados en invernadero en Alajuela, Costa Rica. Las variables cualitativas evaluadas fueron: hábito de crecimiento, ancho de la hoja, espinas en las hojas, color de la corola, largo del fruto, espinas del cáliz del fruto, color del fruto en madurez comercial, y cantidad de semillas. Las variables cuantitativas evaluadas fueron: ancho de hoja $(\mathrm{cm})$, número de espinas por hoja, longitud del fruto (cm), número de espinas por cáliz, y número de semillas por fruto. Se presentaron diferencias significativas entre genotipos para el número de espinas por hoja $(0,04$ para JMX-1099 y 1,44 para JMX-292), longitud del fruto (15,50 y $13,12 \mathrm{~cm}$, respectivamente), número de espinas por cáliz $(0,20$ y 3,48 , respectivamente), y número de semillas por fruto (199,35 y 631,58, respectivamente). Ambos genotipos mostraron características innovadoras para el mercado, como la coloración blanca del fruto del JMX-292, y la poca cantidad de espinas en la hoja y en el cáliz y de semillas en el fruto del JMX-1099, por lo que estos genotipos pueden ser opciones interesantes para los productores.

Palabras clave: color del fruto, cantidad de espinas por hoja, cantidad de espinas por cáliz, número de semillas por fruto.
La berenjena (Solanum melongena L.) pertenece a la familia Solanaceae; presenta una planta herbácea, erecta y ramificada, con el tallo subleñoso, y que sobrepasa el metro de altura cuando está desarrollada (ASERCA, 1999; (Araméndiz, Robles, Cardona, Llano, \& Arzuaga, 2006). La mayoría de los cultivares comerciales de berenjena provienen de cruces de tres variedades botánicas: S. melongena var. esculentum, S. melongena var. serpentinum, y S. melongena var. depressum (Fundación de Desarrollo Agropecuario, 1994).

La berenjena es una hortaliza muy común y apreciada en China, India, Japón, los países del Mediterráneo y los Estados Unidos (ASERCA, 1999). A nivel mundial, se reportó en 2013 una producción de 49 millones de toneladas, y un total de 1,87 millones de hectáreas cultivadas con esta hortaliza, y a China e India como los principales productores (FAO, 2016).

Las berenjenas se caracterizan por la apariencia externa (forma, color y tamaño), sabor, y presencia de semillas. Existe mucha diversidad de genotipos, que varían según el país, la región y el mercado meta. Los frutos poseen diversas formas (esféricas, cilíndricas, ovaladas, ovoides, piriformes, semilargas y largas) y colores (blancas, moradas, verdes, rayadas y negras, entre otras) (ASERCA, 1999; 
Prohens, Muñoz, Rodríguez-Burruezo, \& Nuez, 2005; Kowalska, 2008).

La berenjena es importante en la alimentación pues proporciona sólo $18 \mathrm{kcal} / 100 \mathrm{~g}$ de producto, y posee propiedades beneficiosas para la salud, como laxante, diurético, estimulante de la secreción biliar, y facilita la digestión y ayuda a reducir el índice de colesterol en la sangre (ASERCA, 1999; Antonini, Robles, Neto, \& Kluge, 2002). Además, los frutos son ricos en hierro, potasio, magnesio y calcio (Aminifard, Aroiee, Fatemi, Ameri, \& Karimpour, 2010).

Los descriptores de caracterización propuestos por el Instituto International de Recursos Fitogenéticos (IPGRI, por sus siglas en inglés) para los diferentes cultivos, permiten una discriminación fácil y rápida entre fenotipos, lo cual es importante para describir materiales vegetales de interés comercial. Generalmente se utilizan caracteres altamente heredables, que pueden ser fácilmente detectados a simple vista y que se expresan igualmente en todos los ambientes. Además, se pueden incluir caracteres adicionales que son deseables, según los intereses de los usuarios de un cultivo en particular (IPGRI, 1996). A nivel mundial existe una gran diversidad de variedades de berenjena, y es importante investigar sobre genotipos promisorios, con el fin de encontrar nichos de mercado a nivel nacional e internacional, lo que puede significar una alternativa productiva para el agricultor, y una opción diferenciada de consumo para la población.

En Costa Rica la berenjena se cultiva mayoritariamente en el Valle Central, durante todo el año. Según el Centro Nacional de Abastecimiento y Distribución de Alimentos (CENADA), en 2012 se comercializaron 131 toneladas de berenjena, provenientes principalmente de los cantones de Alfaro Ruiz, Escazú, San Ramón y Paraíso, siendo este último el que reportó la mayor cantidad comercializada con 94,6 \% (PIMA, 2014).

El sistema de producción más utilizado en el cultivo de berenjena en Costa Rica es a campo abierto, pero a nivel mundial se han incorporado los sistemas productivos de ambientes protegidos, como una alternativa limpia de producción que genere una agricultura moderna, competitiva y más saludable, que permita un aumento en el rendimiento y la calidad del fruto, en la extensión de las áreas de producción y los ciclos del cultivo, en la reducción de los daños ocasionados por plagas y enfermedades, en un uso más racional de los agroquímicos, y en una mejor protección del cultivo ante los factores ambientales (Santos, Obregón-Olivas, \& Salamé-Donoso, 2010).

El objetivo de este trabajo fue caracterizar, a nivel morfológico, dos genotipos de berenjena cultivados en invernadero en Alajuela, Costa Rica, con el fin de determinar su potencial comercial para el mercado costarricense.

\section{MATERIALES Y MÉTODOS}

La investigación se desarrolló en la Estación Experimental Agrícola Fabio Baudrit Moreno, de la Universidad de Costa Rica, ubicada en Barrio San José de Alajuela, Costa Rica, a 883msnm, en el periodo comprendido del 14 de agosto de 2013 al 20 de enero de 2014, y se utilizó el invernadero multicapilla del Programa de Hortalizas. Los materiales genéticos de berenjena (Solanum melongena L.) utilizados fueron los híbridos JMX-292 y JMX-1099, provenientes de Estados Unidos.

Para la realización del almácigo, se utilizaron bandejas plásticas de 128 celdas. Se usó una mezcla de fibra de coco y abono orgánico, en proporciones de 2:1, respectivamente. Los híbridos se sembraron en almácigo el 14 de agosto de 2013, y se trasplantaron el 20 de setiembre de ese año; se seleccionaron las plántulas con mejor desarrollo, calidad de adobe, y presencia de tres hojas verdaderas.

Las plántulas fueron trasplantadas en sacos plásticos de $1 \mathrm{~m}$ de largo, $20 \mathrm{~cm}$ de ancho y $18 \mathrm{~cm}$ de alto, rellenos con 30 litros de fibra de coco. Se utilizó el sistema de fertirriego para suplir agua y nutrientes a las plantas.

El genotipo JMX-1099 inició cosecha a los 45 días después del trasplante (ddt), y se extendió por un periodo de 74 días, hasta los 119 ddt. El genotipo JMX-292 inició cosecha a los $55 \mathrm{ddt}$, y se extendió por un periodo de 64 días, hasta los $119 \mathrm{ddt}$. En ambos genotipos, para determinar el momento adecuado de la cosecha, se observaron características propias del fruto, como la coloración brillante, el tamaño, y que el pedúnculo se observara menos suculento.

Se evaluaron cinco variables cuantitativas y nueve variables cualitativas. Las variables cuantitativas fueron:

a. Ancho de hoja (cm): se midió en 144 hojas por genotipo (72 plantas, dos hojas por planta), entre las hojas del tallo principal ubicadas en la parte media de la planta.

b. Número de espinas por hoja: se evaluó en 144 hojas por genotipo (72 plantas, dos hojas por planta), entre las hojas del tallo principal ubicadas en la parte media de la planta.

c. Longitud del fruto $(\mathrm{cm})$ : se midió la longitud de todos los frutos cosechados (710 frutos para el genotipo JMX-1099, y 754 frutos para el JMX-292). 
d. Número de espinas por cáliz: se evaluó en todos los frutos cosechados.

e. Número de semillas por fruto: se evaluó en 120 frutos por genotipo; cada fruto se cortó en cuatro secciones longitudinales, y se extrajeron todas las semillas de la pulpa en forma manual y se contabilizaron.

Para la medición del ancho de hoja y la longitud del fruto, se utilizó una cinta métrica marca Assist, modelo 32G-8025, con una capacidad de 800,0 $\pm 0,1 \mathrm{~cm}$.

Las variables cualitativas se seleccionaron a partir de algunos descriptores para berenjena (Cuadro 1), y fueron las siguientes:

a. Hábito de crecimiento: se evaluó en 12 plantas por genotipo.

b. Ancho de la hoja: se calculó a partir de los datos de las variables cuantitativas.

c. Espinas en las hojas: se calculó a partir de los datos de las variables cuantitativas.

d. Color de la corola: se evaluó en todas las flores de cada planta.

e. Largo del fruto: se calculó a partir de los datos de las variables cuantitativas. f. Espinas del cáliz del fruto: se calculó a partir de los datos de las variables cuantitativas.

g. Color del fruto en madurez comercial: se evaluó en todos los frutos cosechados.

h. Cantidad de semillas: se calculó a partir de los datos de las variables cuantitativas.

Se utilizó un diseño de bloques completos al azar, con tres repeticiones, y cada parcela contó con ocho plantas, de las cuales se evaluaron las seis ubicadas en la posición central de la misma. Para las variables cuantitativas se realizó un análisis de varianza, y se utilizó la prueba de LSD Fisher $(p \leq 0,05)$ para determinar diferencias entre tratamientos.

\section{RESULTADOS}

Para ambos genotipos, el hábito de crecimiento fue erecto, y la mayor parte de las hojas fueron catalogadas como anchas, aunque se presentó alguna variabilidad en esta última característica (Cuadro 2), pues entre un $33 \%$ y $44 \%$ de las mismas presentaron un ancho intermedio, y en el caso del JMX-292 un 1\% de las hojas fueron estrechas.

\section{CUADRO 1}

Descriptores morfológicos utilizados para dos genotipos de berenjena

\begin{tabular}{|c|c|c|}
\hline Carácter & Modalidad & Momento de evaluación \\
\hline Hábito de crecimiento & 3 Erecto; 7 Intermedio & $50 \%$ de las plantas con frutos cosechables \\
\hline Ancho de la hoja & $\begin{array}{l}3 \text { Estrecho }(\sim 10 \mathrm{~cm}) ; 5 \text { Intermedio }(\sim 20 \mathrm{~cm}) \\
7 \text { Ancho }(\sim 30 \mathrm{~cm})\end{array}$ & Posterior a la bifurcación \\
\hline Espinas en las hojas & $\begin{array}{l}0 \text { Ninguna; } 3 \text { Pocas (3-5) } 5 \text { Intermedio (6-10); } \\
7 \text { Muchas }(11-20)\end{array}$ & $\begin{array}{l}50 \% \text { de las plantas con frutos cosechables } \\
45 \mathrm{ddt} \text { para JMX-1099; } 55 \mathrm{ddt} \text { para JMX-292 }\end{array}$ \\
\hline Color de la corola & $\begin{array}{l}1 \text { Blanco verdoso; } 3 \text { Blanco; } 5 \text { Intermedio; } \\
7 \text { Violeta claro; } 9 \text { Violeta azulado }\end{array}$ & 35 ddt para JMX-1099; 40 ddt para JMX-292 \\
\hline Largo del fruto & $\begin{array}{l}5 \text { Intermedio }(\sim 5 \mathrm{~cm}) ; 7 \text { Largo }(\sim 10 \mathrm{~cm}) \text {; } \\
9 \text { Muy largo }(>20 \mathrm{~cm})\end{array}$ & Posterior a la cosecha \\
\hline Espinas del cáliz del fruto & $\begin{array}{l}0 \text { Ninguna; } 3 \text { Pocas }(\sim 5) \\
5 \text { Intermedio }(\sim 10) ; 7 \text { Muchas }(\sim 29)\end{array}$ & Posterior a la cosecha \\
\hline Color del fruto en madurez comercial & $\begin{array}{l}2 \text { Blanca; } 7 \text { Púrpura; } 9 \text { Púrpura negra; } \\
99 \text { Otro (rayada morada y blanca) }\end{array}$ & Posterior a la cosecha \\
\hline Cantidad de semillas & $\begin{array}{l}0 \text { Ninguna; } 2 \text { Muy pocas }(<10) ; 3 \text { Pocas }(\sim 50) \text {; } \\
4 \text { Intermedio }(\sim 100) ; 5 \text { Muchas }(\sim 300)\end{array}$ & Posterior a la cosecha \\
\hline
\end{tabular}

Fuente: (IBPGR, 1988; Araméndiz, Robles, Cardona, Llano, \& Arzuaga, 2006; ECPGR, 2008). 
En ambos casos, la mayoría de los frutos fueron largos, pero la longitud del fruto fue significativamente mayor para el genotipo JMX-1099. En ambos genotipos se presentaron algunos frutos considerados como muy largos.

El color del fruto y de la corola fue diferente para cada genotipo. Además, el JMX-292 presentó una cantidad significativamente mayor de espinas en las hojas y en el cáliz, y de semillas en el fruto, en comparación al genotipo JMX-1099.
El genotipo JMX-292 mostró variabilidad en la cantidad de espinas en las hojas y en el cáliz (desde ninguna a muchas, en ambos casos), mientras que el JMX-1099 presentó variabilidad en la cantidad de espinas en el cáliz (desde ninguna a pocas) y en el número de semillas por fruto (desde ninguna a muchas), y muy poca variabilidad en el número de espinas en la hoja (99\% sin espinas). Todos los frutos del genotipo JMX-292 presentaron muchas espinas.

CUADRO 2

Características de dos genotipos de berenjena.

\begin{tabular}{|c|c|c|c|}
\hline \multirow{2}{*}{ Tipo de variable } & \multirow{2}{*}{ Variable } & \multicolumn{2}{|r|}{ Genotipos } \\
\hline & & JMX-1099 & JMX-292 \\
\hline \multirow[t]{8}{*}{ Cualitativa } & Hábito de crecimiento & $100 \%$ Erecto & $100 \%$ Erecto \\
\hline & Ancho de la hoja & $67 \%$ Ancho; $33 \%$ Intermedio & $55 \%$ Ancho; 44 \% Intermedio; 1 \% Estrecho \\
\hline & Espinas en las hojas & $99 \%$ Ninguna; $1 \%$ Pocas & 75 \% Ninguna; $19 \%$ Pocas; 6 \% Intermedio \\
\hline & Color de la corola & $100 \%$ Intermedio & $100 \%$ Violeta claro \\
\hline & Largo del fruto & 95 \% Largo; 5 \% Muy largo & 99 \% Largo; 1 \% Muy largo \\
\hline & Espinas del cáliz del fruto & $84 \%$ Ninguna; $16 \%$ Pocas & $\begin{array}{l}29 \% \text { Ninguna; } 49 \% \text { Pocas; } 13 \% \text { Intermedio; } \\
9 \% \text { Muchas }\end{array}$ \\
\hline & Color del fruto en madurez comercial & 100 \% Púrpura negra & $100 \%$ Blanco \\
\hline & Cantidad de semillas & $\begin{array}{l}21 \% \text { Ninguna; } 6 \% \text { Muy pocas; } \\
16 \% \text { Pocas; } 12 \% \text { Intermedio; } \\
45 \% \text { Muchas }\end{array}$ & $100 \%$ Muchas \\
\hline \multirow[t]{5}{*}{ Cuantitativa } & Ancho de hoja (cm) & 22,37 a & $21,47 a$ \\
\hline & Número de espinas por hoja & $0,04 \mathrm{a}$ & $1,44 b$ \\
\hline & Longitud del fruto (cm) & $15,50 \mathrm{~b}$ & $13,12 \mathrm{a}$ \\
\hline & Número de espinas por cáliz & $0,20 a$ & $3,48 \mathrm{~b}$ \\
\hline & Número de semillas por fruto & 199,35 a & $631,58 \mathrm{~b}$ \\
\hline
\end{tabular}

* Letras distintas indican diferencias significativas $(p \leq 0,05)$, según la prueba LSD Fisher.

\section{DISCUSIÓN}

Hábito de crecimiento: El hábito de crecimiento de ambos genotipos fue erecto. Este comportamiento fue similar al obtenido por otros investigadores al caracterizar 13 variedades de berenjena (Araméndiz et al., 2006), lo que evidencia que es una característica que ha sido incorporada preferentemente en los procesos de mejoramiento genético de esta especie.

Color de la corola: Para el genotipo JMX-1099, el color de la corola fue intermedio, es decir, de coloración blanca y morada. Por otra parte, para el JMX-292, la corola fue de color violeta claro, lo cual es similar a lo obtenido por otro autor con otro genotipo de berenjena blanca (Criado, 2012). Esta cualidad fue muy uniforme en cada genotipo ( $100 \%$ de las plantas en cada caso), y se considera una característica definida y estable.
Color del fruto en madurez comercial: El color es un indicador de la calidad gustativa, la cual está determinada por la intensidad del mismo, que a su vez condiciona la calidad de fruto (Nuez, 2001).

Los frutos de color externo negro-púrpura, tales como los del genotipo JMX-1099, dominan el mercado, y corresponden a los que contienen antocianinas en su cáscara. Existen también variedades de color blanco, como el JMX-292, cuya cáscara carece de dichos pigmentos (Kowalska, 2008; Passam \& Karapanos, 2008).

Varios autores mencionan que cada vez hay mayor interés y demanda en los mercados europeos por nuevos tipos de berenjena, de colores diferentes al negro intenso (como el color blanco), y también por los tipos mini, especialmente adaptados a los usos de familias de uno o dos miembros (Prohens et al., 2005). 
Los caracteres de color de corola y de fruto son variables cualitativas, propias de cada genotipo, gobernadas por genes mayores y con mínima influencia del ambiente. El genotipo JMX-292 podría ser considerado más innovador que el JMX-1099, ya que comercialmente es diferente a lo que el consumidor está acostumbrado a conseguir en el mercado, debido al color del fruto.

Es muy importante aprender a reconocer el punto de cosecha adecuado de la berenjena, pues no existe un rasgo evidente que lo indique, a diferencia de otras solanáceas como chile y tomate, en los cuales la cosecha está definida por un cambio en la coloración del fruto.

Ancho de la hoja: Para ambos genotipos, la mayoría de hojas se ubicaron en la categoría de hojas anchas. En otro ensayo con 13 variedades de berenjena, la mayoría de las hojas presentó un valor intermedio para esta característica (Araméndiz et al., 2006), aunque también se presentaron hojas anchas, al igual que lo obtenido en el presente trabajo. A nivel cuantitativo, no se presentaron diferencias significativas entre genotipos para esta característica.

Número de espinas en la hoja: Casi la totalidad (99\%) de las hojas evaluadas en el genotipo JMX-1099 no presentaron espinas. En el caso del JMX-292, se encontró una gran variabilidad con respecto a esta característica, pues un $19 \%$ de las hojas presentó pocas espinas, y un $6 \%$ mostró un nivel intermedio de ellas.

El genotipo JMX-1099 presentó una cantidad significativamente menor de espinas en las hojas que el JMX292 (0,04 y 1,44 espinas/hoja, respectivamente). Aunque esta es una característica que no influye sobre la calidad del fruto, sí influye sobre el manejo agronómico; la ausencia de espinas en las hojas y en el cáliz facilita las labores del cultivo, especialmente la cosecha (Araméndiz et al., 2006).

Longitud del fruto: El genotipo JMX-1099 presentó frutos significativamente más largos $(15,50 \mathrm{~cm})$ que el JMX-292 (13,12cm), por lo que esta característica se debe tener en cuenta al considerar los distintos nichos de mercado y los gustos del consumidor.

Existe mucha variabilidad en berenjena con respecto a la longitud del fruto, según el genotipo evaluado. Diversos investigadores han hallado que la longitud del fruto puede oscilar entre 10,7 y 23,9 cm (Pérez, Montoya, Cardona, Araméndiz, \& Robles, 2006; Macua, 2007; Maldaner et al., 2009; Criado, 2012; Torres, 2013), los resultados obtenidos en el presente trabajo se ubican dentro de dicho rango.

En Brasil, se considera que los frutos de primera calidad deben tener una longitud superior a $17 \mathrm{~cm}$, y los de segunda calidad entre 13 y $17 \mathrm{~cm}$, y aquellos con menos de $13 \mathrm{~cm}$ se consideran no comerciales (Antonini et al., 2002). Sin embargo, en Costa Rica se toma en cuenta el peso del fruto (y no su longitud) como criterio de calidad comercial (J. E. Monge-Pérez, datos sin publicar).

Número de espinas en el cáliz del fruto: La cantidad de espinas en el cáliz del fruto es una característica muy importante a nivel del manejo poscosecha, ya que un fruto con gran cantidad de ellas puede provocar inconvenientes para su manipulación, por lo que es preferible que la cantidad de espinas sea poca o ninguna. Considerado lo anterior, se recomienda el genotipo JMX-1099, ya que presentó una cantidad significativamente menor de espinas en el cáliz (0,20 espinas/cáliz; $84 \%$ de los cálices sin espinas), con respecto al JMX-292 (3,48 espinas/cáliz; $71 \%$ de los cálices tiene de pocas a muchas espinas).

Se debe resaltar la mayor variabilidad del genotipo JMX-292 en comparación al JMX-1099, con respecto a esta variable, pues presentó un $29 \%$ de los cálices sin espinas, $49 \%$ con pocas espinas, $13 \%$ con un nivel intermedio de espinas, y $9 \%$ con muchas espinas. En un ensayo anterior se encontró que un genotipo de berenjena blanca presentó 12,2 espinas/cáliz (Criado, 2012), lo que es superior a los resultados obtenidos en el presente ensayo.

Una característica que se busca en las variedades híbridas modernas de berenjena es que no presenten espinas en el cáliz ni en otras partes de la planta (Prohens et al., 2005).

Número de semillas en el fruto: Esta es una característica muy importante a nivel del fruto, pues entre mayor sea la cantidad de semillas, mayor el sabor amargo de la pulpa, y esto podría ser una limitante del cultivo a nivel comercial, ya que en muchas ocasiones el consumidor lo rechaza debido al sabor. Esta es una característica asociada a la variedad y no al cultivo propiamente, por lo que es recomendable un fruto con pocas semillas para lograr una mejor comercialización del mismo.

Para el caso de los híbridos estudiados en el presente ensayo, se recomienda el genotipo JMX-1099, ya que presentó una cantidad significativamente menor de semillas en el fruto (199,35 semillas/fruto; sólo un $45 \%$ de los frutos con muchas semillas), con respecto 
al JMX-292 (631,58 semillas/fruto; $100 \%$ de los frutos tienen muchas semillas).

El genotipo JMX-1099 presentó una alta variabilidad en esta característica, la cual podría deberse a efectos ambientales, pues los frutos con pocas semillas (16\%), muy pocas semillas (6\%), o ninguna semilla (21\%), se obtuvieron principalmente en las primeras cosechas (inicios de noviembre 2013), mientras que la mayoría de los frutos con muchas semillas (45\%) se obtuvieron en las cosechas de diciembre 2013.

La presencia de un alto número de semillas en el fruto de berenjena no es deseable para el consumidor, ya que afecta adversamente la calidad (Khah, 2005). La presencia de glicoalcaloides en el fruto de berenjena, especialmente a altas concentraciones, puede causar sabor amargo (Passam \& Karapanos, 2008).

La reducción en el número de semillas del fruto de berenjena es un factor positivo de calidad, ya que las semillas se endurecen conforme el fruto madura, y esto puede provocar sabor amargo, debido al aumento en el contenido de fenoles asociados con ellas en el tejido de la placenta; esto se da particularmente cuando los frutos se dejan demasiado tiempo en la planta (Passam \& Karapanos, 2008). Esto enfatiza la importancia de realizar la cosecha en el momento oportuno.

En berenjena, aunque la polinización, fertilización y la presencia de semillas son procesos requeridos para el normal cuajado y desarrollo del fruto, la presencia de semillas maduras reduce la calidad del fruto, tanto para consumo fresco como para proceso. Las semillas hacen el fruto menos palatable ya que la pulpa se endurece, inducen un más rápido y más intenso oscurecimiento de la pulpa luego del cortado, y aumentan las concentraciones de saponina y solasonina, que causan sabor amargo (Karapanos, Mahmood, \& Thanopoulos, 2008).

El número de semillas en el fruto depende, entre otros aspectos, de la longitud del estilo de la flor a partir del cual se forma el fruto. En berenjena existen cuatro longitudes del estilo: largo, mediano, corto, y pseudo-corto; en un estudio se encontró que los frutos provenientes de flores de estilo corto presentaron menos semillas (entre 141,0 y 217,80 semillas/fruto) que los provenientes de flores de estilo mediano o largo (entre 358,5 y 376,5 semillas/fruto) (Sekara \& Bieniasz, 2008). Las flores de estilo corto son particularmente comunes durante la época con mayores temperaturas, lo que produce una reducción en el cuajado del fruto y el rendimiento (Karapanos, Mahmood, \& Thanopoulos, 2008), y también podría producir una menor cantidad de semillas en el fruto (Sekara \& Bieniasz, 2008).
La frecuencia con que se encuentran los cuatro tipos de flores en berenjena es una característica que depende del genotipo; todos los tipos de longitud del estilo están presentes en la misma planta, y aún dentro del mismo racimo (Karapanos et al., 2008). Además del factor genético, la longitud del estilo en berenjena también está influenciado por factores externos, como la cantidad de frutos que tiene la planta en un momento determinado, la edad de la planta, y las condiciones ambientales (Karapanos et al., 2008; Kowalska, 2008; Sekara \& Bieniasz, 2008. Las temperaturas bajas afectan la viabilidad y germinación del polen en berenjena, lo que produce frutos con pocas semillas o sin ellas (Passam \& Karapanos, 2008).

Las flores en berenjena pueden aparecer solitarias, en racimos, o mixtas (tanto solitarias como en racimos). En los racimos, la flor "basal" está bien desarrollada, posee estilo largo o mediano, y es funcionalmente fértil, pero las otras flores son más pequeñas, y muchas de ellas son funcionalmente estériles pues tienen un estilo de longitud reducida (estilo corto o pseudo-corto) (Karapanos et al., 2008).

En berenjena, la formación de semillas es máxima en flores en que el estigma, en su madurez, está situado cerca de los poros de las anteras (Sekara \& Bieniasz, 2008). La heterostilia (diferencia en la posición del estigma en relación a la parte superior del cono formado por las anteras) es un fenómeno común en esta especie, lo cual se debe principalmente a la ocurrencia de estilos de diferente longitud, mientras que la longitud de los estambres no varía (Karapanos et al., 2008).

En un ensayo se demostró que un mismo genotipo de berenjena produjo una menor cantidad de semillas en sus frutos al ser cultivado en invernadero (entre 769,2 y 842,5 semillas/fruto), en comparación a la siembra a campo abierto (entre 909,1 y 1226,9 semillas/fruto) probablemente debido a un mayor nivel de polinización entomófila en las condiciones de campo (Khah, 2005; 2011); esto constituye una de las ventajas de la producción de berenjena en invernadero.

Se concluye que el genotipo JMX-1099 presentó varias características favorables, como la poca cantidad de espinas en la hoja y el cáliz, y la baja cantidad de semillas en el fruto, lo que facilita el manejo agronómico y poscosecha del cultivo, y brinda un fruto "dulce" (no amargo), con mayor atractivo para el consumidor. Por otra parte, el genotipo JMX-292 presentó frutos de color blanco, lo que representa un producto innovador en el mercado.

Entre los caracteres estudiados en el presente trabajo, el hábito de crecimiento, el color de la corola, y el color del fruto en madurez comercial, mostraron una alta estabilidad, mientras que el ancho de la hoja, el largo del 
fruto, el número de espinas en la hoja y en el cáliz, y la cantidad de semillas en el fruto, presentaron una variabilidad importante, influenciados probablemente por factores ambientales y fisiológicos.

\section{AGRADECIMIENTOS}

Los autores agradecen la colaboración de Laura Soto, María José Moya, Julio Vega, Andrés Oviedo y Juan Manuel Ávalos por el apoyo brindado en el trabajo de campo, y de Mario Monge en la traducción del resumen al idioma inglés. Asimismo, agradecen el financiamiento recibido por parte de la Vicerrectoría de Investigación de la Universidad de Costa Rica.

\section{REFERENCIAS}

Aminifard, M. H., Aroiee, H., Fatemi, H., Ameri, A., \& Karimpour, S. (2010). Responses of eggplant (Solanum melongena L.) to different rates of nitrogen under field conditions. Journal of Central European Agriculture, 11(4), 453-458. doi: 10.5513/JCEA01/11.4.863

Antonini, A. C., Robles, W. G., Neto, J. T., \& Kluge, R. A. (2002). Capacidade produtiva de cultivares de berinjela. Horticultura Brasileira, 20(4), 646-648. doi: 10.1590/ S0102-05362002000400027

Araméndiz, H., Robles, J. R., Cardona, C. E., Llano, J. D., \& Arzuaga, E. A. (2006). Caracterización morfológica de la berenjena (Solanum melongena L.). Temas Agrarios, 11(1), 5-14.

ASERCA. (1999). La berenjena, una hortaliza desconocida en nuestro país, pero con enorme vocación exportadora. Revista Claridades Agropecuarias(72), 3-17.

Criado, A. (2012). Caracterización de variedades locales de berenjena y judía en Esporus, Centro de Conservación de la Biodiversidad Cultivada. Barcelona, España: Universidad de Barcelona.

ECPGR. (2008). Minimum descriptors for eggplant, Capsicum (sweet and hot pepper) and tomato. European Cooperativa Programme for Plant Genetic Resources.

FAO. (2016). Estadísticas de mercado de berenjena a nivel mundial. Recuperado de www.fao.org

Fundación de Desarrollo Agropecuario. (1994). Cultivo de berenjena. Santo Domingo, República Dominicana: Centro para el Desarrollo Agropecuario y Forestal.

IBPGR. (1988). Descriptors for eggplant. Roma, Italia: International Board for Plant Genetic Resources.

IPGRI. (1996). Descriptores para el cultivo del tomate (Lycopersicon spp.). Roma, Italia: International Plant Genetic Resources Institute.

Karapanos, I. C., Mahmood, S., \& Thanopoulos, C. (2008). Fruit set in solanaceous vegetable crops as affected by floral and environmental factors. The European Journal of Plant Science and Biotechnology, 2(Special Issue 1), 88-105.

Khah, E. M. (2005). Effect of grafting on growth, performance and yield of aubergine (Solanum melongena L.) in the field and greenhouse. Journal of Food, Agriculture \& Environment, 3(3\&4), 92-94.

Khah, E. M. (2011). Effect of grafting on growth, performance and yield of aubergine (Solanum melongena L.) in greenhouse and open-field. International Journal of Plant Production, 5(4), 359-366.

Kowalska, G. (2008). Flowering biology of eggplant and procedures intensifying fruit set - review. Acta Scientiarum Polonorum Hortorum Cultus, 7(4), 63-76.

Macua, J. I. (2007). Influencia de la densidad de plantación en el cultivo de la berenjena. XXXVI Seminario de Técnicos y Especialistas en Horticultura (pp. 87-91). Ibiza: Centro de Publicaciones Agrarias, Pesqueras y Alimentarias, Ministerio de Agricultura, Alimentación y Medio Ambiente.

Maldaner, I. C., Guse, F. I., Streck, N. A., Heldwein, A. B., Lucas, D. D., \& Loose, L. H. (2009). Filocrono, área foliar e produtividade de frutos de berinjela conduzidas com uma e duas hastes por planta em estufa plástica. Ciência Rural, 39(3), 671-677. 10.1590/S0103-84782009005000013

Nuez, F. (2001). El cultivo del tomate; anatomía y fisiología de la planta. Bilbao, España: Ediciones Mundi-Prensa.

Passam, H. C., \& Karapanos, I. C. (2008). Eggplants, peppers and tomatoes: factors affecting the quality and storage life of fresh and fresh-cut (minimally processed) produce. The European Journal of Plant Science and Biotechnology, 2(Special Issue 1), 156-170.

Pérez, M., Montoya, R. A., Cardona, C. E., Araméndiz, H., \& Robles, J. R. (2006). Efecto de cuatro densidades de población sobre el crecimiento del fruto de berenjena (Solanum melongena L.). Temas Agrarios, 11(2), 14-25.

PIMA. (2014). Estadísticas sobre la comercialización de berenjena en el CENADA, Costa Rica. Heredia, Costa Rica: Sistema de Información de Mercados Mayoristas, Programa Integral de Mercadeo Agropecuario.

Prohens, J., Muñoz, J. E., Rodríguez-Burruezo, A., \& Nuez, F. (2005, octubre 15). Últimos avances en la mejora genética de la berenjena. Vida Rural, 52-56.

Santos, B., Obregón-Olivas, H., \& Salamé-Donoso, T. (2010). Producción de hortalizas en ambientes protegidos: estructuras para la agricultura protegida. Recuperado de http://edis.ifas.ufl.edu/pdffiles/HS/HS118200.pdf

Sekara, A., \& Bieniasz, M. (2008). Pollination, fertilization and fruit formation in eggplant (Solanum melongena L.). Acta Agrobotanica, 61(1), 107-113. doi: 10.5586/ aa.2008.014

Torres, M. A. (2013). Evaluación de frecuencia y dos concentraciones de aplicación de bioestimulante (Biozyme) en floración de berenjenas (Solanum melongena L.) para reducir los costos del cultivo. Santiago, Chile: Facultad de Ciencias Agronómicas, Universidad de Chile. 